\title{
Influence of indenter tip roundness on hardness behavior in nanoindentation
}

\author{
Weimin Chen ${ }^{\mathrm{a}}$, Min $\mathrm{Li}^{\mathrm{b}}$, Taihua Zhang ${ }^{\mathrm{c}}$, \\ Yang-Tse Cheng ${ }^{\mathrm{d}}$, Che-Min Cheng ${ }^{\mathrm{c}, *}$ \\ ${ }^{a}$ Division of Engineering Science and Research, Institute of Mechanics, CAS, Beijing 100080, China \\ ${ }^{\mathrm{b}}$ School of Aeronautics Sciences and Technology, Beijing University of Aeronautics and Astronautics, Beijing 100083, China \\ ${ }^{\mathrm{c}}$ State Key Laboratory for Nonlinear Mechanics, LNM, Institute of Mechanics, CAS, Beijing 100080, China \\ ${ }^{\mathrm{d}}$ Materials and Processes Laboratory, General Motors Research and Development Center, Warren, MI 48090, USA
}

Received 1 May 2006; received in revised form 2 September 2006; accepted 14 September 2006

\begin{abstract}
In this paper, the effect of indenter tip roundness on hardness behavior for two typical elastic perfectly plastic materials is studied by means of finite element simulation. A rigid conical indenter of semi apex angle $70.3^{\circ}$ fitted smoothly with a spherical tip is employed. It is shown that as the indentation depth increases hardness first rises from zero, reaches a maximum and then decreases slowly approaching asymptotically the limiting value equal to that due to a conical indenter of ideally sharp tip. The range within which hardness varies appreciably is comparable to the radius of the indenter tip. The difference between the maximum value and the limiting value depends on the yield stress over the Young's modulus ratio. The smaller this ratio the greater the difference is. Numerical simulation also provides an opportunity for checking the accuracy and limitations of the widely used Oliver-Pharr method.
\end{abstract}

(C) 2006 Elsevier B.V. All rights reserved.

Keywords: Indenter tip roundness; Indentation size effect; The Oliver-Pharr method

\section{Introduction}

Instrumented nanoindentation has become an important and popular tool for measuring the mechanical property of materials at micro- and nanometer length scales. For elastic plastic materials, it readily yields values of hardness and the Young's modulus. If such a material is also homogeneous and if the indenter is geometrically self-similar in shape hardness will be independent of indentation depth. Commercially available indenters such as the Berkovich and the Vickers indenters may be considered geometrically self-similar if tip rounding is small enough to be ignored. Experimentally, it is found that hardness is indeed a constant when the indentation depth is sufficiently large.

For small indentation depths, it is well known that hardness is no longer a constant for most elastic plastic materials. In fact, it often increases as the indentation depth decreases from a few hundred nanometers to several tens of nanometers. An improved understanding of this indentation-size effect is the goal of many recent investigations. There are good reasons to believe that at

\footnotetext{
* Corresponding author. Tel.: +86 1062553654 ; fax: +86 1062561284 .

E-mail address: zhengzm@imech.ac.cn (C.-M. Cheng).
}

this length scale many materials may no longer be regarded as homogeneous and without internal structure. This consideration has led to much fruitful research in recent years highlighted by the strain gradient theory [1-4]. On the other hand since the tip radius of indenters is known to be about $100 \mathrm{~nm}$ or more, its effect on the apparent hardness can no longer be ignored [5-7]. Since these two effects are simultaneously present [8-17] in hardness measurement there is a need to separate the two possible causes, one physical and the other geometrical, in a quantitative way.

This paper examines, by means of finite element simulation, the hardness behavior of the homogeneous and elastic perfectly plastic material as a function of indentation depth using a rigid conical indenter fitted smoothly with a spherical cap. The aim is to establish a quantitative basis upon which other factors contributing to the indentation size effect can be separately studied.

Section 2 of this paper describes the mathematical model of the present problem. Section 3 presents the principal numerical results of the finite element simulation. In Section 4, these results are used to analyze the widely employed Oliver-Pharr method for obtaining hardness and Young's modulus from instrumented measurements. Conclusions are given in the final section. 


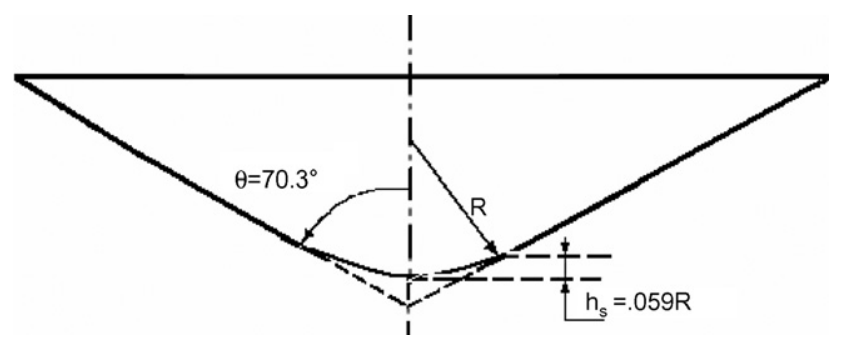

Fig. 1. The ideal indenter-conic indenter with a spherical cap.

\section{The mathematical model}

The body of the indenter used in the finite element simulation is assumed to be conical with semi apex angle, $\vartheta$, equal to $70.3^{\circ}$. This angle is chosen so that the cone with a sharp tip would have the same volume to height relation as that of the ideally sharp Berkovich and Vickers indenter. In order to simulate a blunt indenter, the cone is fitted smoothly with a spherical cap of radius $R$. This geometry of the conical indenter is shown schematically in Fig. 1. It is furthermore assumed to be rigid. Mathematically, the contact area $A$ can be expressed explicitly in terms of the contact depth $h_{\mathrm{c}}$ in the following manner,

$\frac{A}{\pi R^{2}}=\frac{h_{\mathrm{c}}}{R}\left(2-\frac{h_{\mathrm{c}}}{R}\right)$, if $\frac{h_{\mathrm{c}}}{R} \leq 1-\sin \vartheta$

$\frac{A}{\pi R^{2}}=\left(\tan \vartheta\left(\frac{h_{\mathrm{c}}}{R}+\frac{1-\sin \vartheta}{\sin \vartheta}\right)\right)^{2}$, if $\quad \frac{h_{\mathrm{c}}}{R} \geq 1-\sin \vartheta$

when $\vartheta=70.3^{\circ}, 1-\sin \vartheta=0.059$. Transition from contact with the spherical head to contact with the cone takes place at $h_{\mathrm{c}}=0.059 R$.

The material being simulated is assumed to be isotropic, homogeneous and elastic perfectly plastic (hardening exponent $n=0$ ). Two typical cases are studied. The yield stress over Young's modulus ratio, $\sigma_{\mathrm{Y}} / E$, of one material is 0.1 . This material exhibits sink-in behavior. The other material has a $\sigma_{\mathrm{Y}} / E$ ratio equal to 0.003 and exhibits pile-up behavior. For both materials the Poison ratio $v$ is taken to be 0.3 .

Dimensional analysis $[18,19]$ shows that hardness $H$ is related to indentation depth $h$ in the following way,

$H=E h^{2} f\left(\frac{h}{R}, \frac{\sigma_{Y}}{E}, v, n, \vartheta\right), \quad \vartheta=70.3^{\circ}, \quad n=0$

and the contact depth is related to the indentation depth in a similar way,

$\frac{h_{\mathrm{c}}}{R}=g\left(\frac{h}{R}, \frac{\sigma_{\mathrm{Y}}}{E}, v, n, \vartheta\right)$

The indentation depth corresponding to $h_{\mathrm{c}} / R=0.059$ will be denoted by $h_{\mathrm{s}}$. For indentation depth smaller and greater than $h_{\mathrm{s}}$, the contact area is, respectively, given by the first and second of Eq. (1).

\section{Dependence of hardness on indentation depth}

Finite element simulation is executed using ABQUAS [20], and the results of hardness behavior for the two materials are

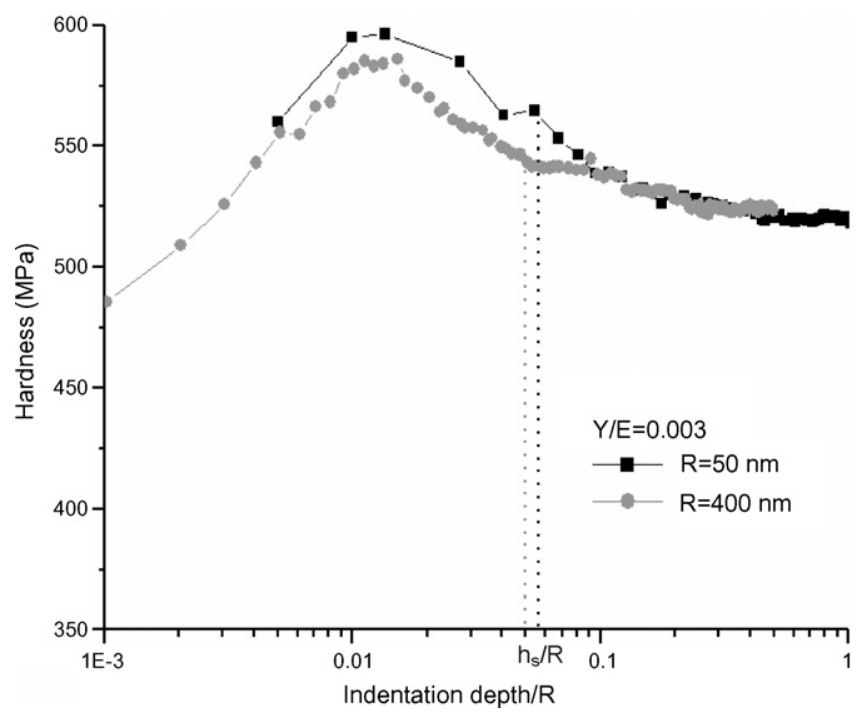

Fig. 2. Computed hardness vs. reduced indentation depth $h / R$ using the ideal indenter for $\sigma_{\mathrm{Y}} / E=0.003$ and $n=0$.

shown in Figs. 2 and 3. In each case, numerical computation was carried out for two values of the tip radius, namely 50 and $400 \mathrm{~nm}$. We see from the figures that for each material the two sets of results nearly collapse into a single curve as required by Eq. (2). The reason that they do not exactly do so is due to numerical error.

The hardness behavior of the two materials has two common features. Firstly, as the indentation depth increases from zero hardness increases and reaches a maximum value. It then decreases and approaches asymptotically a limiting value equal to the hardness due to an ideally sharp conical indenter. Secondly, the range in terms of the indentation depth within which the hardness varies appreciably is quite large and is of the order of the indenter tip radius.

The hardness behavior of the two materials also differs in several ways. Relative to the hardness at large indentation depth

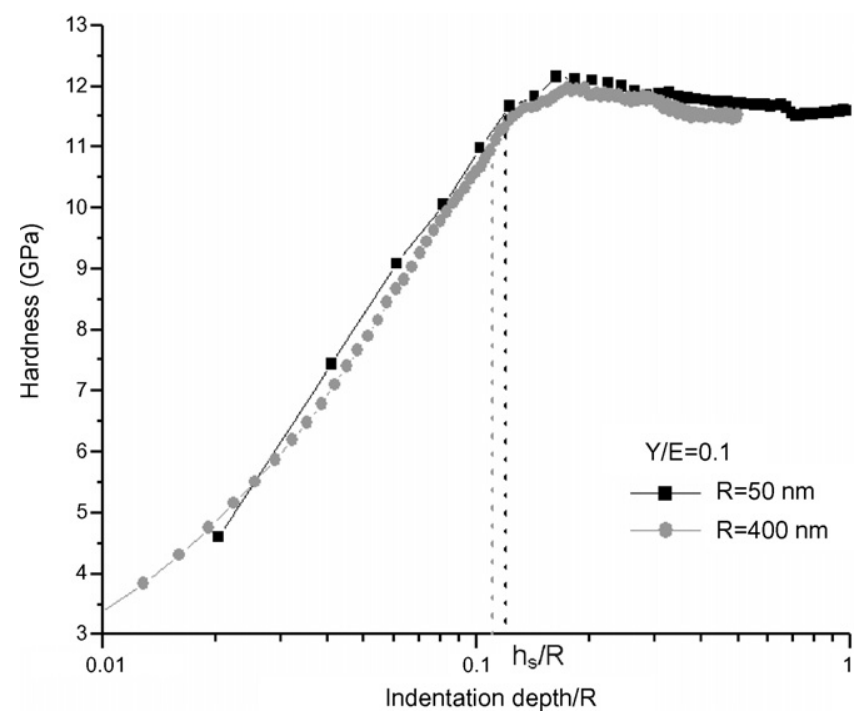

Fig. 3. Computed hardness vs. reduced indentation depth $h / R$ using the ideal indenter for $\sigma_{\mathrm{Y}} / E=0.3$ and $n=0$. 
the peak value of hardness for the material with a low value of $\sigma_{\mathrm{Y}} / E$ is, percentage wise, larger than for the material with a high value of $\sigma_{\mathrm{Y}} / E$. In other words, scale effect due to tip roundness is more pronounced for materials with low values of relative yield stress. In Figs. 2 and 3, the points where the indentation depth $h$ equals to $h_{\mathrm{s}}$ are marked. We note that for the material with lower relative yield stress the peak hardness occurs at an indentation depth smaller than $h_{\mathrm{s}}$, meaning that this takes place while the spherical tip is in contact with the material. For the material with higher relative yield stress peak hardness occurs at an indentation depth greater than $h_{\mathrm{s}}$ so that the contact is with the conical body of the indenter.

The initial increase of hardness with indentation depth is consistent with the experimental observation reported by Lee et al. [21]. Since for materials with low values of yield stress the maximum hardness is reached at an indentation depth smaller than $h_{\mathrm{s}}=0.059 R \approx 10 \mathrm{~nm}$, this part of the hardness versus indentation depth curve may easily be missed in hardness measurements.

\section{Application}

As an application, finite element simulations is used to examine the Oliver-Pharr [22] method for instrumented indentation measurements. Calculation of hardness using finite element simulation is a "direct" problem where one calculates hardness from known material properties and geometry. On the other hand, the determination of hardness and Young's modulus is an inverse problem where one deduces hardness and Young's modulus from measured load versus indentation depth relation for loading and unloading. It is under such circumstances that the Oliver-Pharr method is used. Since this method makes use of semi-empirical formulae, it is of interest to see to what extent these formulae can be justified at small indentation depth. Because the method is not designed for materials where pile-up occurs around indents, the following discussion is limited to sink-in cases.

From the loading and unloading curves, one obtains the peak load $P$ and the initial unloading slope $S=(\mathrm{d} P / \mathrm{d} h)_{\mathrm{u}}$ at the point of unloading as functions of the indentation depth $h$. Then, the contact depth $h_{\text {cop }}$ is, according to the Oliver-Pharr method,

$h_{\text {cop }}=h-\frac{P}{S} \frac{2(\pi-2)}{\pi}$

The contact area is next calculated using the area function,

$A_{\mathrm{op}}=c_{0} h_{\mathrm{cop}}^{2}+\sum_{i=1}^{N} c_{i} h_{\mathrm{cop}}^{1 / 2^{i-1}}, N=8$ or less

where $c_{0}$ and $c_{i}$ are known constants determined through a calibration process. Hardness $H$ and Young's modulus $E$ then follow from the definition of hardness and another formula

$$
\begin{aligned}
& H_{\mathrm{op}}=\frac{P}{A_{\mathrm{op}}} \\
& E_{\mathrm{op}}=\frac{\sqrt{\pi}\left(1-v^{2}\right)}{2 \beta} \frac{S}{\sqrt{A_{\mathrm{op}}}}
\end{aligned}
$$

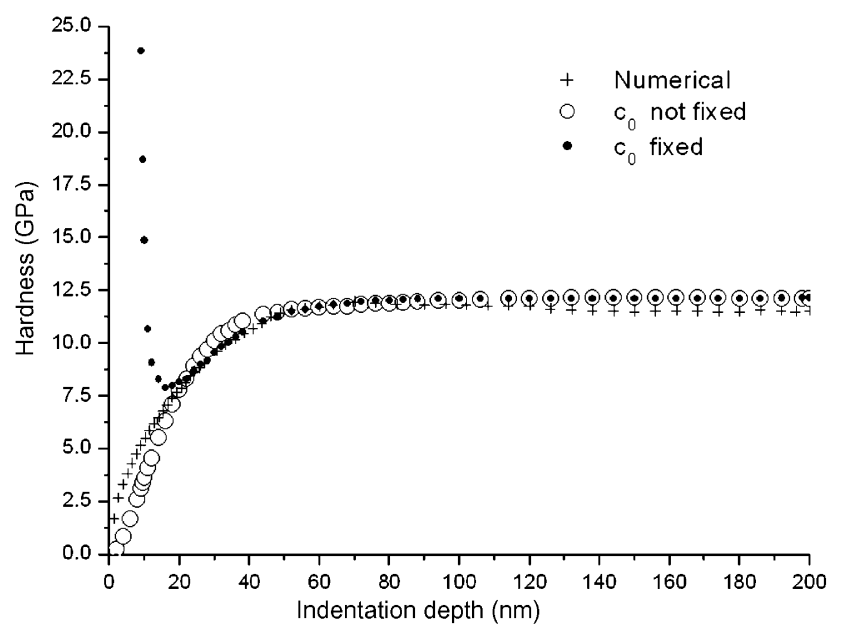

Fig. 4. $H_{\text {op }}$ vs. $h$ for $h_{\max }=200 \mathrm{~nm}$.

where $\beta$ is an empirical constant. Relation (7) with $\beta=1.0$ has been justified theoretically for infinitesimal deformation and $\beta>1$ is introduced to count for large deformation [23]. It is usually set at 1.05-1.08 [23]. The subscript op implies that the relevant quantity is obtained using the Oliver-Pharr method.

To establish the area function for a specific indenter, the loading and unloading curves of a standard material (usually fused silica) are measured. One calculates $h_{\text {cop }}$ as a function of $h$ using Eq. (4). Since for a standard material both $E$ and $v$ are known a priori, Eq. (7) can be used to calculate the contact area $A_{\mathrm{op}}$ (after replacing $E_{\text {op }}$ by $E$ ) also as an explicit function of $h$. In this way, one obtains a set of values of ( $h_{\mathrm{cop}}, A_{\mathrm{op}}^{*}$ ) for a set of values of $h($ or $P$ ). The best fit of the area function Eq. (5) to this set of values then determines the unknown coefficients $c_{0}$ and $c_{i}$. It is known that values of these coefficients depend also on the range of indentation depth (or of load) as well as on whether additional constraints are put on the coefficients (such as setting $c_{0}$ to 24.5 to make the area function approach asymptotically the contact area of a conic indenter with $\vartheta=70.3^{\circ}$ at large indentation depths).

First, we check the overall performance of the Oliver-Pharr method. Using the loading and unloading data from the finite element simulation as input as if they were given experimentally, we establish the area function for the ideal indenter defined by Eq. (1) by following the recipe of the Oliver-Pharr method. Using the same set of data and the contact area $A_{\mathrm{op}}$ thus obtained we calculate hardness $H_{\mathrm{op}}$ and Young's modulus $E_{\mathrm{op}}$ as function of $h$ by means of Eqs. (6) and (7). The overall performance of this method can then be quantitatively evaluated by (a) how well this hardness agrees with the hardness $H$ obtained by finite element simulation and (b) how well the calculated Young's modulus agrees with the value $E$ assigned $a$ priori.

Figs. 4 and 5 compare $H_{\mathrm{op}}$ and $H$ for a range of $h$ equals to 200 and $2500 \mathrm{~nm}$, and for two choices of $c_{0}$. The material properties are: $\sigma_{\mathrm{Y}} / E=0.1, v=0.3, E=70 \mathrm{GPa}$, and indenter tip radius $R=400 \mathrm{~nm}$. We note that $H_{\mathrm{op}}$ deviates considerably from $H$ at small indentation depth. Moreover, the curves $H_{\text {op }}$ versus $h$ at small indentation depth may behave quite differently for different choices of the indentation depth range and $c_{0}$. In fact 


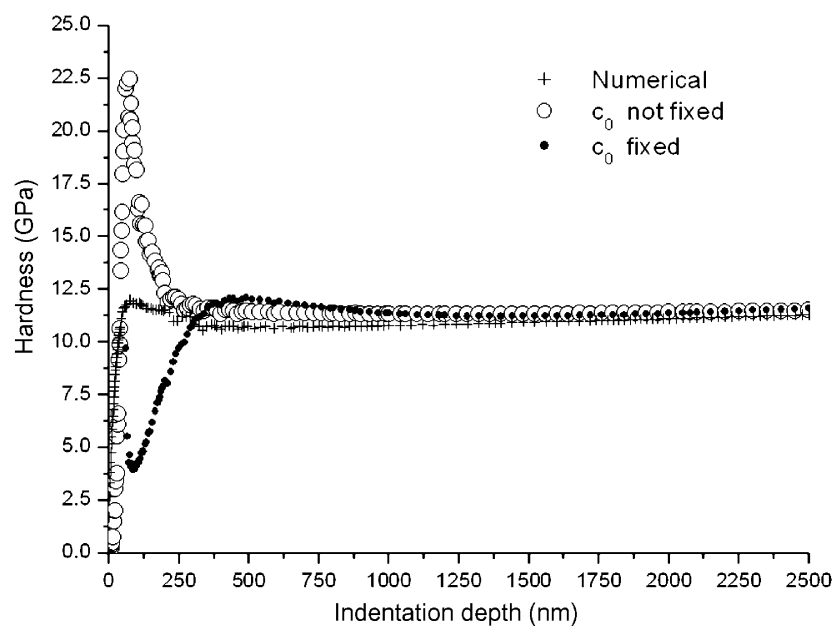

Fig. 5. $H_{\text {op }}$ vs. $h$ for $h_{\max }=2500 \mathrm{~nm}$.

for certain choices, this method may over-predict hardness by a factor much larger than unity. Fig. 6 contrasts $E_{\text {op }}$ with $E$ in a similar manner. Again, deviations at small indentation depth are quite noticeable. Figs. 4-6 demonstrate that the Oliver-Pharr method may not be accurate at small indentation depth.

Next, we show how and why such inaccuracy arises. Numerical simulation provides values of $P, S, h_{\mathrm{c}}, A$ for a given $h$. The differences of corresponding quantities from the simulation and the Oliver-Pharr results are regarded as errors. We calculate the contact depth $h_{\text {cop }}$ by means of Eq. (4). Then a measure of the error in contact depth due to the application of Eq. (4) is simply $h_{\text {cop }} / h$. Similarly the error due to the application of Eq. (7) in calculating the contact area $A_{\mathrm{op}}^{*}$ can be expressed as $A_{\mathrm{op}}^{*} / A$. An additional error is introduced when fitting the data set $\left(h_{\text {cop }}, A_{\mathrm{op}}^{*}\right)$ by a smooth curve to obtain a reconstructed area function $A_{\mathrm{op}}\left(h_{\mathrm{cop}}\right)$. A measure of the error in curve fitting is $A_{\text {op }}\left(h_{\text {cop }}\right) / A_{\text {op }}^{*}\left(h_{\text {cop }}\right)$. Since Eq. (4) defines a one-to-one correspondence between indentation depth and contact depth, we can also replace $A_{\text {op }} / h_{\text {cop }}$ by $A_{\text {op }}(h)$. However, we note that transforming $A_{\mathrm{op}}\left(h_{\mathrm{cop}}\right)$ to $A_{\mathrm{op}}(h)$ introduces an additional error due to the use Eq. (4).

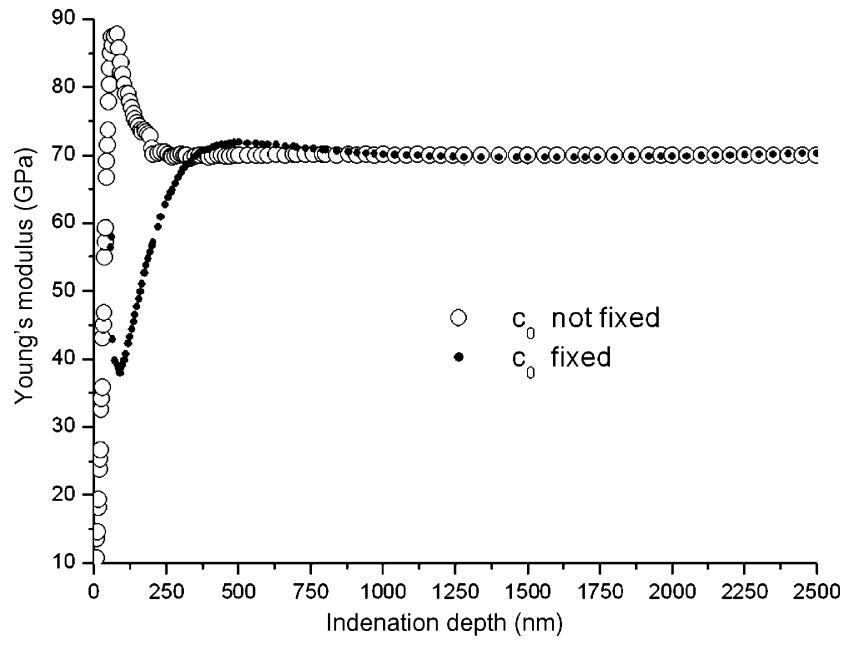

Fig. 6. Young's modulus vs. indentation depth, $h_{\max }=2500 \mathrm{~nm}$.

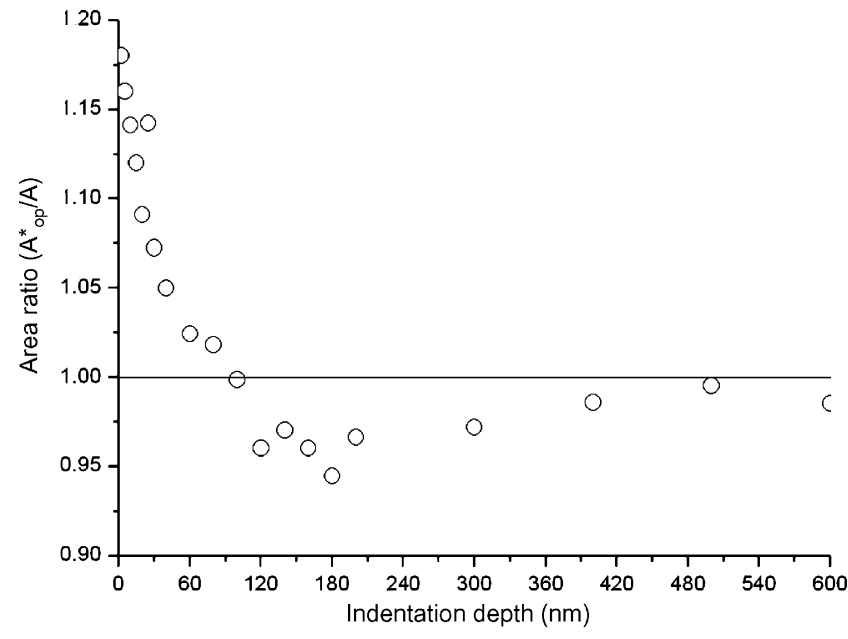

Fig. 7. Area ratio $\left(A_{\mathrm{OP}}^{*} / A\right)$ vs. indentation depth.

In our example, the Oliver-Pharr method returns a value of Young's modulus $E_{\mathrm{op}}(h)$ different from $E$. It is easily shown from the definition of $A_{\mathrm{op}}^{*}$ and $E$ that

$E_{\mathrm{op}}(h) \sqrt{A_{\mathrm{op}}(h)}=E \sqrt{A_{\mathrm{op}}^{*}(h)}$

Consequently, $E_{\mathrm{op}} / E$ versus $h$ is a direct measure of the error due to the fitting process. Fig. 6 indicates that curve fitting by means of the area function, Eq. (5), can be poor at small indentation depth (note that $E$ is a constant).

Since by definition $A H(h)=A_{\mathrm{op}}(h) H_{\mathrm{op}}(h)=P$, we arrive at another relation.

$\frac{H_{\mathrm{op}}}{H}=\frac{A}{A_{\mathrm{op}}}=\frac{A}{A_{\mathrm{op}}^{*}} \frac{A_{\mathrm{op}}^{*}}{A_{\mathrm{op}}}=\left(\frac{E_{\mathrm{op}}}{E}\right)^{2} \frac{A}{A_{\mathrm{op}}^{*}}$

In this relation, $A_{\mathrm{op}}^{*} / A$ is a measure of the error in $A_{\mathrm{op}}^{*}$ due to the use of Eq. (7) $(\beta=1.08)$. Fig. 7 plots $A_{\mathrm{op}}^{*} / A$ against $h$. It shows that this ratio can differ significantly from one at small indentation depth. We know that Eq. (7) at small indentation depth is only accurate for $\beta=1.0$ and that the evaluation of the unloading slope $S$ at small indentation depth can involve large error.

In Fig. $8, h_{\text {cop }} / h_{c}$ representing errors due to the use of Eq. (4) is plotted against $h$. Like in the previous figures, the error appears to grow as $h$ decreases toward zero. This is expected since Eq. (4), a generalization of a relation in the elastic penetration by an ideally sharp cone, is not applicable to a spherical indenter.

The above analysis shows that all three kinds of errors become more prominent as the indentation depth approaches zero. To examine why this happens we discuss the behavior of $A_{\mathrm{op}}$ and $H_{\text {op }}$ near $h=0$. For our ideal indenter we know that at the limit $h_{\mathrm{c}}=0$, to the first order of approximation, $A \approx 2 \pi R h_{\mathrm{c}} \propto h_{\mathrm{c}}$. Finite element simulation as well as analysis shows that $H \rightarrow 0$ at this limit. Then, the definition of hardness requires that the load $P \propto h_{\mathrm{c}}^{m}, m>1$ as $h_{\mathrm{c}} \rightarrow 0$. Likewise, for a homogeneous material the unloading slope $S$ must behave like $S \propto h_{\mathrm{c}}^{1 / 2}$ in order to maintain a finite Young's modulus. Let us define $\delta A=A_{\mathrm{op}}-A$ as the error in the contact area, then $\delta A \propto c_{N} h_{\mathrm{c}}^{1 / 2^{N-1}}$. Conse- 


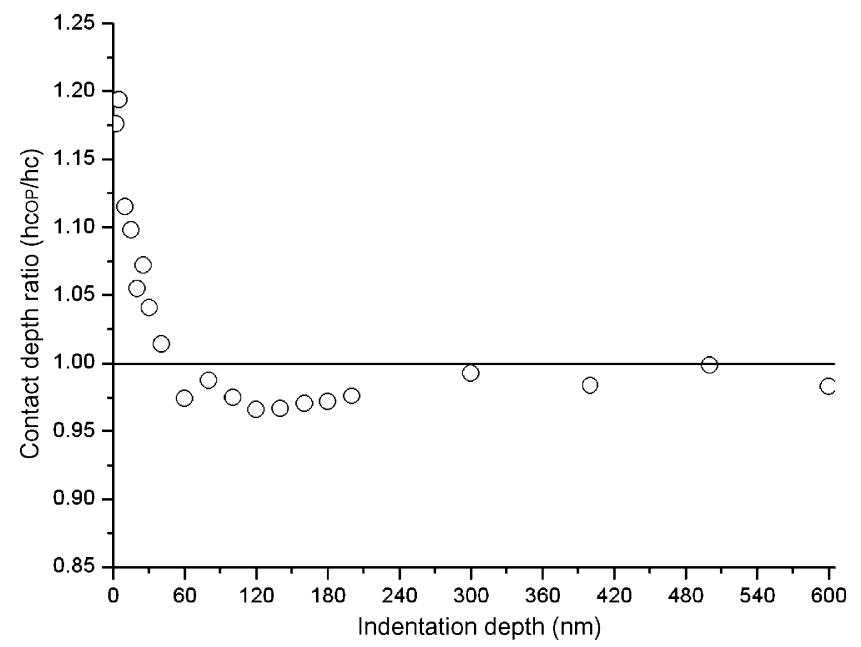

Fig. 8. Contact depth ratio vs. indentation depth.

quently, as $h_{\mathrm{c}} \rightarrow 0$, we have $H_{\mathrm{op}}=P / A_{\mathrm{op}}=P / A+\delta A \approx P / \delta A$. This means that there always exists a certain region close to $h_{\mathrm{c}}=0$ where the hardness calculated according to the Oliver-Pharr method is dominated by error in the calculation of contact area, resulting in unreliable hardness and Young's modulus (for example, a negative $c_{N}$ would lead to a negative contact area sufficiently close to $h_{\mathrm{c}}=0$ ). Since the value of coefficients in the area function depends on many factors such as the quality and quantity of raw data, the range of contact depth within which fitting is done, whether or not constraints on the constants $c_{0}$ and $c_{i}$ are put in place, etc., the behavior of the hardness curve near $h_{c}=0$ becomes unreliable. For the same reason the size of this region is subject to variations.

Thus, although the Oliver-Pharr method yields reliable hardness and Young's values at sufficiently large indentation depth (roughly greater than $R$, Figs. 4-6) when the material is elastic perfectly plastic, the above analysis shows that at very small indentation depth (about $0.059 R$ and less) the method is likely to produce spurious results. The main reason is that in this region (a) the contact area is very small making it difficult to reliably calculate hardness and Young's modulus, (b) data are sparse (poor fitting), (c) the error in Eq. (4) is large and (d) the analytical form of the area function is a poor representation of contact area. Errors incurred at such small indentation depth can be carried into region beyond $0.059 R$ because of the smoothness requirement of the fitting curve. How far this region extends seems to depend on many factors including the calibration process, quality and quantity of initial data (Figs. 4 and 5).

\section{Concluding remarks}

1. For elastic perfectly plastic materials, tip rounding can cause appreciable increase in hardness at indentation depth comparable to the tip radius. Decreasing the ratio $\sigma_{\mathrm{Y}} / E$ makes this kind of "scale effect" more pronounced. The peak value of hardness for $\sigma_{\mathrm{Y}} / E=0.003, \nu=0.3$ and $E=70 \mathrm{Gpa}$ is approximately $13 \%$ higher than its limiting value at large indentation depth.
2. Tip rounding causes hardness to vary with indentation depth. The range in terms of the indentation depth within which hardness varies significantly is roughly on the order of $R$.

3. For sink-in elastic perfectly plastic materials, the Oliver-Pharr method offers a useful tool. With proper choice of the range of indentation depth or load in the calibration of the area function, it leads to accurate values of hardness and Young's modulus at indentation depth large relative to the indenter tip radius. But, care must be exercised at small indentation depth. As the indentation depth approaches zero, the method may yield erroneous hardness and Young's values mainly because the contact area approaches zero and the Oliver-Pharr method may not provide accurate values for the contact depth or area. This error may be a contributing factor to indentation size effect in nanometer scales.

4. To properly interpret indentation data at small indentation depth it is desirable that the hardness and Young's modulus data be examined together with specific information regarding calibration of the related area function, because the latter information may help identify probable error contained in the hardness and Young's modulus measurements.

\section{Acknowledgement}

The authors would like to acknowledge the support from the National Science Foundation of China, project nos. 10372101 and 10432050 .

\section{References}

[1] M.R. Begley, J.W. Hutchinson, J. Mech. Phys. Solids 46 (1998) 20492068.

[2] W.D. Nix, H. Gao, J. Mech. Phys. Solids 46 (1998) 411-425.

[3] Y. Huang, Z. Xue, H. Gao, W.D. Nix, Z.C. Xia, J. Mater. Res. 15 (2000) $1786-1796$.

[4] Y.G. Wei, J.W. Hutchinson, J. Mech. Phys. Solids 51 (2003) 2037-2056.

[5] K.W. McElhaney, J.J. Vlassak, W.D. Nix, J. Mater. Res. 13 (1998) 1300-1306.

[6] J.G. Swadener, E.P. George, G.M. Pharr, J. Mech. Phys. Solids 50 (2002) 681-694.

[7] N. Iwashita, M.V. Swain, Phil. Mag. A. 82 (2002) 2199-2205.

[8] W.D. Nix, Mater. Trans. 29A (1989) 2217-2245.

[9] M.S. de Guzman, G. Neubauer, P.A. Flinn, W.D. Nix, Mater. Res. Symp. Proc. 308 (1993) 613-618.

[10] N.A. Stelmashenko, A.G. Walls, L.M. Brown, Y.V. Milman, Acta Metall. Mater. 41 (1993) 2855-2865.

[11] M. Atkinson, J. Mater. Res. 10 (1995) 2908-2915.

[12] Q. Ma, D.R. Clarke, J. Mater. Res. 10 (1995) 853-863.

[13] W.J. Poole, M.F. Ashby, N.A. Fleck, Scr. Mater. 34 (1996) 559-564.

[14] S. Suresh, T.G. Nieh, B.W. Choi, Scr. Mater. 41 (1999) 951-957.

[15] A.V. Zagrebelny, E.T. Lilleodden, W.W. Gerberich, C.B. Carter, J. Am. Ceram. Soc. 82 (1999) 1803-1809.

[16] G. Feng, W.D. Nix, Scr. Mater. 51 (2004) 599-603.

[17] R. Saha, Z. Xue, Y. Huang, W.D. Nix, J. Mech. Phys. Solids 49 (2001) 1997-2014.

[18] Y.T. Cheng, C.M. Cheng, Mater. Sci. Eng. R. 44 (2004) 91-149.

[19] Y.T. Cheng, C.M. Cheng, Int. J. Solids Struct. 36 (1999) 1231-1243.

[20] HKS, Inc., Pawtucket, RI, USA.

[21] K.W. Lee, Y.W. Chung, C.Y. Chan, Surf. Coat. Technol. 168 (2003) 57-61.

[22] W.C. Oliver, G.M. Pharr, J. Mater. Res. 7 (1992) 1564-1583.

[23] W.C. Oliver, G.M. Pharr, J. Mater. Res. 19 (2004) 3-20. 